\title{
Hybridizing wood cellulose and graphene oxide toward high-performance fibers
}

\author{
Yuanyuan $\mathrm{Li}^{1,2,4}$, Hongli $\mathrm{Zhu}^{2,4}$, Shuze $\mathrm{Zhu}^{3,4}$, Jiayu Wan ${ }^{2}$, Zhen $\mathrm{Liu}^{2}$, Oeyvind Vaaland ${ }^{2}$, Steven Lacey ${ }^{2}$, \\ Zhiqiang Fang ${ }^{2}$, Hongqi Dai ${ }^{1}$, Teng $\mathrm{Li}^{3}$ and Liangbing $\mathrm{Hu}^{2}$
}

High-performance microfibers such as carbon fibers are widely used in aircraft and wind turbine blades. Well-aligned, strong microfibers prepared by hybridizing two-dimensional (2D) graphene oxide (GO) nanosheets and one-dimensional (1D) nanofibrillated cellulose (NFC) fibers are designed here for the first time and have the potential to supersede carbon fibers due to their low cost. These well-aligned hybrid microfibers are much stronger than microfibers composed of 1D NFC or 2D GO alone. Both the experimental results and molecular dynamics simulations reveal the synergistic effect between GO and NFC: the bonding between neighboring GO nanosheets is enhanced by NFC because the introduction of NFC provides the extra bonding options available between the nanosheets. In addition, 1D NFC fibers can act as 'lines' to 'weave and wrap' 2D nanosheets together. A 2D GO nanosheet can also bridge several 1D NFC fibers together, providing extra bonding sites between 1D NFC fibers over a long distance. The design rule investigated in this study can be universally applied to other structure designs where a synergistic effect is preferred.

NPG Asia Materials (2015) 7, e150; doi:10.1038/am.2014.111; published online 9 January 2015

\section{INTRODUCTION}

Strong synthetic fibers such as carbon fibers have an important role in a range of applications from aircraft to wind turbine blades. However, these fibers are expensive and demonstrate limited performance. Nanofibrillated cellulose (NFC), derived mainly from wood, is an inexhaustible one-dimensional (1D) material with a diameter in nanoscale and a length in microscale. ${ }^{1}$ This material has been explored as a possible building block for high-strength composites due to its impressive mechanical properties with an elastic modulus of $\sim 140$ $\mathrm{GPa}^{2}$ Moreover, NFC possesses a high specific area and strong interacting surface hydroxyls, and can therefore act as an excellent reinforcement/binder. ${ }^{3,4}$ In addition, chemically exfoliated twodimensional (2D) graphene oxide (GO) nanosheets exhibit excellent mechanical properties, a high aspect ratio and good processibility, making the nanosheets another attractive building block to produce strong microfibers. ${ }^{5}$ Numerous hydroxyl, epoxide functional groups and carboxyl groups are present on the GO nanosheet basal planes and edges, providing strong bonding sites and allowing the GO nanosheets to be well-dispersed in water. ${ }^{6}$ Transforming $2 \mathrm{D}$ GO nanosheets into strong and highly ordered microfibers is a promising feat. ${ }^{7-12}$ Efforts have been made to improve the mechanical properties of GO-based microfibers by chemical cross-linking and polymer coatings as well as through giant GO nanosheets. ${ }^{13-16}$ GO microfibers exhibiting a tensile strength of $442 \mathrm{MPa}$ and an elastic modulus of $47 \mathrm{GPa}$ have been reported. ${ }^{10,11}$ The performance, especially the mechanical strength, must be further improved for practical use. ${ }^{17}$

In this study, we design and investigate micro-sized fibers by hybridizing 2D GO and 1D NFC. Great synergistic interactions between 2D GO and 1D NFC are identified, which led to a noticeable improvement in the mechanical properties of these microfibers compared with microfibers made solely from one of the two building blocks. The underlying hypothesis is that the 1D flexible NFC fibers have good interfacial contact with the 2D GO nanosheets and enhance the bonding between the 2D GO nanosheets. In addition, the 1D NFC fibers can act as 'lines' to 'weave and wrap' 2D nanosheets together. 2D GO nanosheets can create a bridge between the neighboring NFC fibers, providing extra bonding sites between 1D NFC fibers over a long distance. Molecular dynamics simulations clearly verify this hypothesis and demonstrate that this synergistic interaction can enhance both the tensile strength and toughness of the microfibers. The synergistic interaction between 1D NFC and 2D GO revealed in this study is also applicable to other material building blocks, facilitating a new design strategy to create a wide range of mechanically strong microfibers. Figure 1 shows the structure of the GO-NFC hybrid microfibers produced in this study. In the hybrid microfiber, the GO nanosheets and NFC fibers align along the direction of the microfiber. Metal ions $\left(\mathrm{Ca}^{2+}\right)$ link the NFC and GO nanosheets together to further enhance the tensile strength of the hybrid microfiber.

${ }^{1}$ College of Light Industry Science and Engineering, Nanjing Forestry University, Nanjing, Jiangsu, P.R. China; ${ }^{2}$ Department of Materials Science and Engineering, University of Maryland, College Park, MD, USA and ${ }^{3}$ Department of Mechanical Engineering, University of Maryland, College Park, MD, USA

${ }^{4}$ These authors contributed equally to this work.

Correspondence: Professor H Dai or Professor T Li or Professor L Hu, Materials Science and Engineering, University of Maryland College Park, 1208 Engineering Lab Building, College Park, MD 20742, USA.

E-mails: binghu@umd.edu or lit@umd.edu or daihq@njfu.com.cn

Received 11 September 2014; revised 2 October 2014; accepted 2 October 2014 

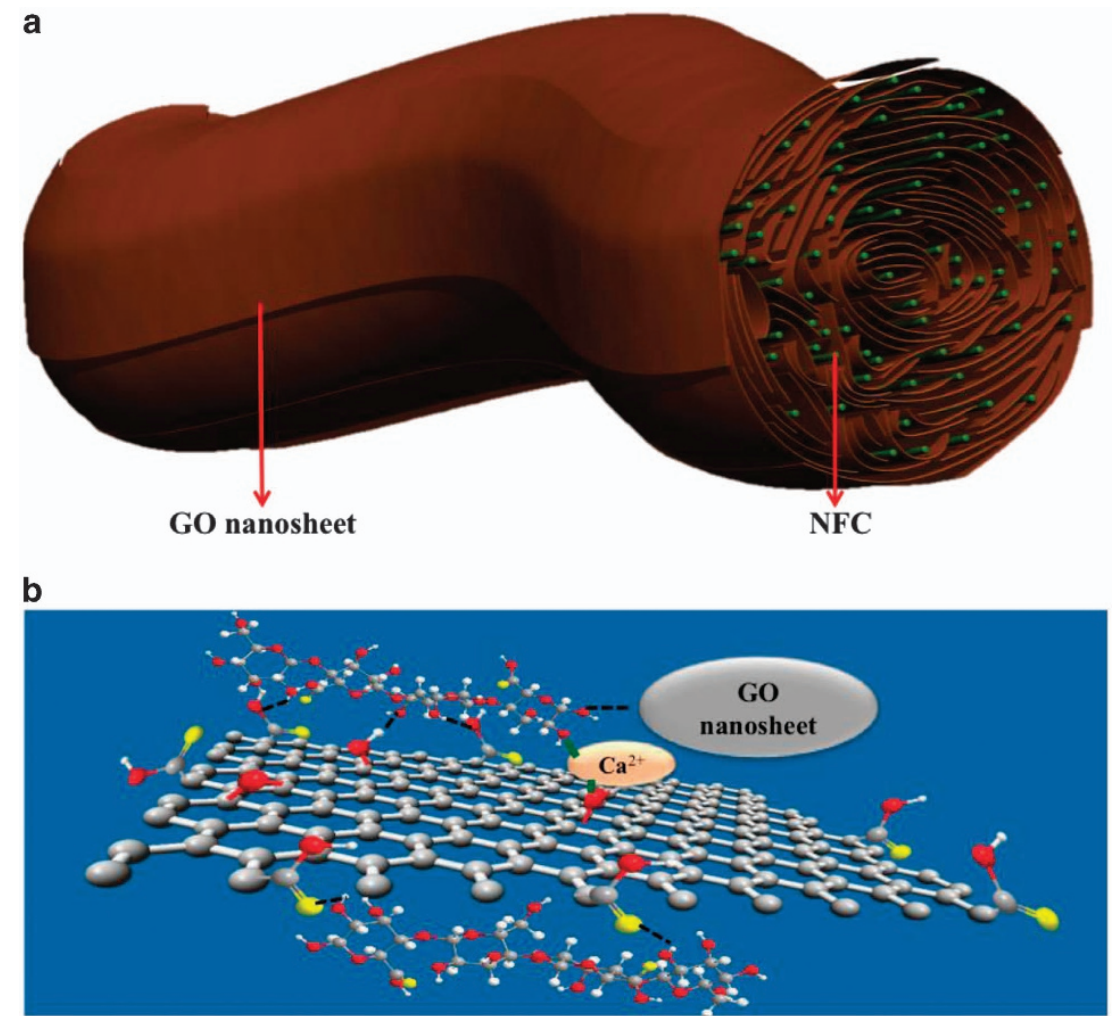

Figure 1 (a) Schematic of a GO-NFC hybrid microfiber consisting of aligned GO nanosheets and NFC fibers along the microfiber direction. The synergistic interaction between NFC and GO leads to greatly improved mechanical strength, elastic modulus and toughness. (b) Structural representation of how metal ion $\left(\mathrm{Ca}^{2+}\right)$ infiltration further increases the bonding between building blocks in the hybrid microfiber. GO, graphene oxide; NFC, nanofibrillated cellulose.

\section{EXPERIMENTAL PROCEDURE}

\section{Preparation of materials}

The NFC was prepared according to the literature. ${ }^{18}$ First, $78 \mathrm{mg}(2,2,6,6-$ tetramethylpiperidin-1-yl)oxidanyl (TEMPO), $514 \mathrm{mg}$ sodium bromide ( $\mathrm{NaBr}$ ), and $5 \mathrm{~g}$ Kraft bleached softwood pulp were mixed together. The TEMPO oxidation of the cellulose was initiated by adding $30 \mathrm{ml}$ of $12 \% \mathrm{NaClO}$ at room temperature under gentle agitation. During the oxidation process, the $\mathrm{pH}$ was maintained at 10.5. After the TEMPO treatment, the fibers were thoroughly washed with distilled water and disintegrated by one pass through a $\mathrm{M}-110 \mathrm{EH}$ microfluidizer (Microfluidics Industries, Newton, MA, USA) to obtain a NFC suspension.

The GO was prepared according to Hummer's method. ${ }^{19}$ Graphite flakes ( $3.0 \mathrm{~g}, 1$ wt. equiv.) and $\mathrm{NaNO}_{3}$ (1.5 g, 0.5 wt. equiv.) were mixed together and cooled to $0{ }^{\circ} \mathrm{C} . \mathrm{H}_{2} \mathrm{SO}_{4}(98 \%, 69 \mathrm{ml})$ was added to the mixture and stirred uniformly before $\mathrm{KMnO}_{4}(9.0 \mathrm{~g}, 3$ wt. equiv.) was added slowly. The reaction temperature was maintained below $20^{\circ} \mathrm{C}$ while adding the $\mathrm{KMnO}_{4}$; then, the reaction was warmed to $35^{\circ} \mathrm{C}$ and stirred for $30 \mathrm{~min}$. Next, water $(138 \mathrm{ml})$ was added slowly to the mixture while maintaining the reaction temperature at $98^{\circ}$ $\mathrm{C}$ for $15 \mathrm{~min}$. The mixture was then allowed to cool, and additional water $(420 \mathrm{ml})$ and $30 \% \mathrm{H}_{2} \mathrm{O}_{2}(3 \mathrm{ml})$ were added. After cooling in air, the mixture was purified by washing with distilled water in a filter. A GO cake was obtained, and an aqueous GO solution was then formed after sonicating for $30 \mathrm{~min}$.

Wet spinning of the GO-NFC hybrid microfiber was conducted by hand. The GO-NFC hybrid solution (1.1 wt \%) in water was extruded directly into ethanol, and the fiber was pulled out to dry in air after $1 \mathrm{~min}$ of immersion. A force was applied at the ends of the fibers to allow the fibers to dry under tension. After drying, the fiber was infiltrated in a $10 \mathrm{wt} \% \mathrm{CaCl}_{2}$ water solution for $1 \mathrm{~h}$. The infiltrated GO-NFC hybrid microfibers were attained by pulling the fibers out of the solution, and drying them in air under tension. The GO microfibers were prepared by extruding $1.1 \mathrm{wt} \% \mathrm{GO}$ solution into $1 \mathrm{wt} \% \mathrm{NaOH}$ in ethanol to ensure the formation of GO microfibers. Note that GO will disperse in ethanol such that the fibers will not form in a coagulation bath of pure ethanol. The NFC microfibers were prepared by extruding a concentrated 1.6 wt $\%$ NFC solution into ethanol before drying the fibers in air under tension.

The tensile strength of the fibers was tested using a dynamic mechanical analysis machine (Q800, TA Instruments, New Castle, DE, USA) in tension mode. Before the test, each sample was conditioned for $24 \mathrm{~h}$ at $50 \%$ humidity at $\sim 23^{\circ} \mathrm{C}$. Each fiber was $20 \mathrm{~mm}$ long, and the strain ramp was set at $5 \% \mathrm{~min}^{-1}$.

\section{Simulation methodology}

The full atomistic simulations used the ReaxFF potential ${ }^{20}$ as implemented in the Large-scale Atomic/Molecular Massively Parallel Simulator ${ }^{21}$ simulation package, developed for carbon-carbon interactions and hydrocarbon oxidation. A first-principles-based ReaxFF force field was developed and was able to account for various non-bonded interactions, including an explicit expression for hydrogen bonds. ${ }^{22}$ In molecular dynamics models for GO sheets with and without NFC, the carbon atoms on the left and right end of the GO sheet were confined to only have the degree of freedom along the sliding direction. The vertical separation distance between the left ends or right ends was fixed to be $0.7 \mathrm{~nm}$ (about the typical inter-layer distance between GO sheets) for GO with NFC and $0.8 \mathrm{~nm}$ for GO without NFC. The system was equilibrated at $300 \mathrm{~K}$ in canonical ensemble before a constant translational velocity of $0.0002 \AA / \mathrm{fs}$ was assigned to the right end of the top GO sheet. The left end of the bottom GO sheet was held in position. The force was calculated by summing the force components along the sliding direction of all the atoms in the top GO sheet. In molecular dynamics models for NFC chains with and without GO nanosheets, the carbon atoms on the sinistral of the left NFC chains were fixed in motion while the carbon atoms on the dextral of the right NFC chains were confined to only have the degree of freedom along the sliding direction. The system was equilibrated at $300 \mathrm{~K}$ in canonical ensemble before a constant translational velocity of $0.0004 \AA /$ fs was assigned to the right end of the dextral NFC chains. The force was calculated by summing the force components along the sliding 
direction of all the atoms in the two dextral NFC chains. In all the simulations, the time step was $0.25 \mathrm{fs}$ to ensure the stability of the simulations.

\section{RESULTS AND DISCUSSION}

The GO nanosheets used in this study were prepared using Hummer's method. ${ }^{19}$ The lateral size of the GO nanosheet was $\sim 1.5 \mu \mathrm{m}$, as shown in Figure 2a. Characterization of the GO nanosheets by atomic force microscopy (AFM) demonstrated that the average size of the sheets was $1.2 \mu \mathrm{m}$. The prepared GO demonstrated good solubility in water (Figure 2a) and formed liquid crystal (LC) at a concentration of $1.1 \mathrm{wt} \%$ (Supplementary Figure S1a). NFCs were obtained by the TEMPO oxidation of the wood fibers followed by one pass through a microfluidizer. The diameter of the NFC was $\sim 10 \mathrm{~nm}$, and the length was 100-400 nm (Figure 2b). TEMPO-oxidized nanocelluloses tend to self-assemble and form a chiral nematic phase at an appropriate concentration. $^{23}$ In our study, the NFC solution formed weak birefringence at a concentration of $1.0 \mathrm{wt} \%$, indicating the onset of ordering (Supplementary Figure S1b).

In addition to the excellent mechanical properties of the individual building blocks themselves, the alignment and interaction between the building blocks are crucial to achieve superior mechanical properties. ${ }^{24}$ Alignment is a proven strategy to achieve high-strength nanocomposites by providing the maximum packing density with the minimum amount of defects for crack formation and propagation. ${ }^{25}$ Both concentrated NFC and GO suspensions can self-assemble into ordered LC arrangements; this methodology has been demonstrated to create strong and well-aligned fibers. ${ }^{13,26,27}$ Here, we prepared a LC spinning solution consisting of GO and NFC (weight ratio of 1:1) at a concentration of $1.1 \mathrm{wt} \%$ (Figure 2c). When GO and NFC were uniformly mixed together, the solution yielded a uniform parallelbanded texture that was distinct from pure GO as the width of the aligned bands was thinner than the GO LC (Figure $2 \mathrm{c}$ and Supplementary Figure S1a). After drying, substantial alignment was observed (as depicted in Figure 2d), which supports the LC phase of the spinning solution.

The mechanically strong hybrid microfibers were prepared by extruding the GO-NFC spinning solution directly into a coagulation bath of ethanol. A skin was induced when the spinning solution was extruded into ethanol, and therefore, a gel microfiber formed. Note that a solvent exchange from water to ethanol occurred in the coagulation bath. ${ }^{28}$ After the gel fiber was pulled out of the bath, the ethanol evaporated to form a dry hybrid microfiber. Figure 3a shows four hybrid microfibers that were prepared simultaneously by extruding the spinning solution into ethanol. From $1 \mathrm{ml}$ of spinning solution, fibers that are more than $10 \mathrm{~m}$ long can be produced (Figure 3b). Diameters between $10-40 \mu \mathrm{m}$ can be created and tuned by changing the syringe needles with different diameters. The hybrid microfibers were strong and flexible and could be readily twisted into yarn by hand (Figure 3c).

To improve the fiber alignment and strength, the hybrid microfibers were dried under tension, causing the building blocks to align along the fiber direction. During the drying process, the fiber diameter
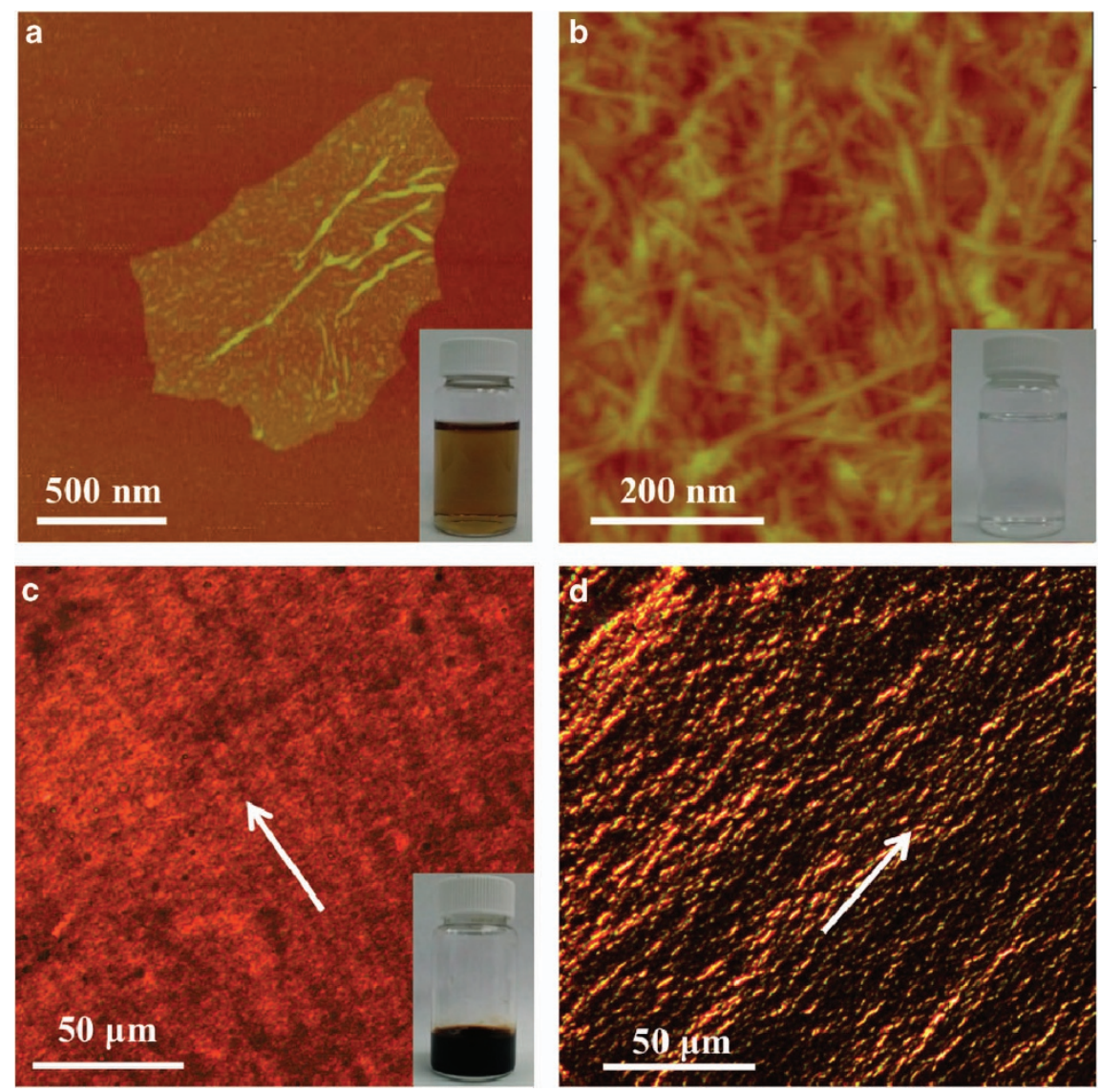

Figure 2 (a) AFM image of a GO nanosheet; the inset is an optical image of the GO nanosheet solution. (b) AFM image of NFC; the inset is an optical image of the NFC solution. (c) Polarized optical microscopy (POM) image of the GO-NFC liquid crystal solution showing a typical LC phase; the inset is an optical image of the GO-NFC solution used for wet spinning. (d) POM image of the GO-NFC solution after drying, which shows clear alignment. AFM, atomic force microscopy; GO, graphene oxide; LC, liquid crystal; NFC, nanofibrillated cellulose. 


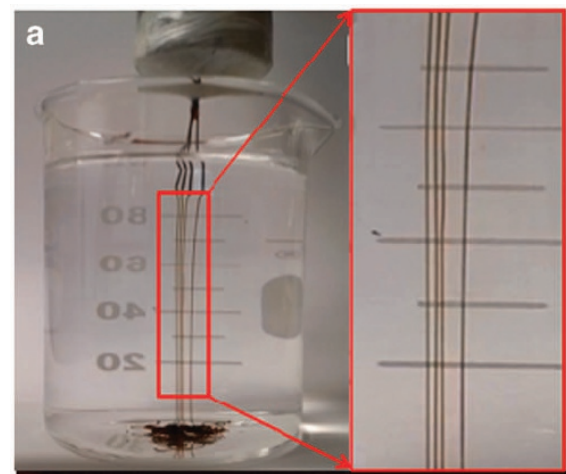

d

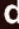

$50 \mu \mathrm{m}$

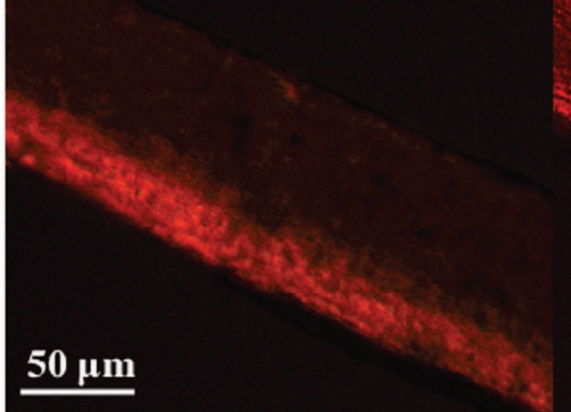

\section{b}
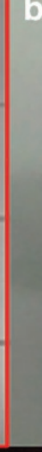

e
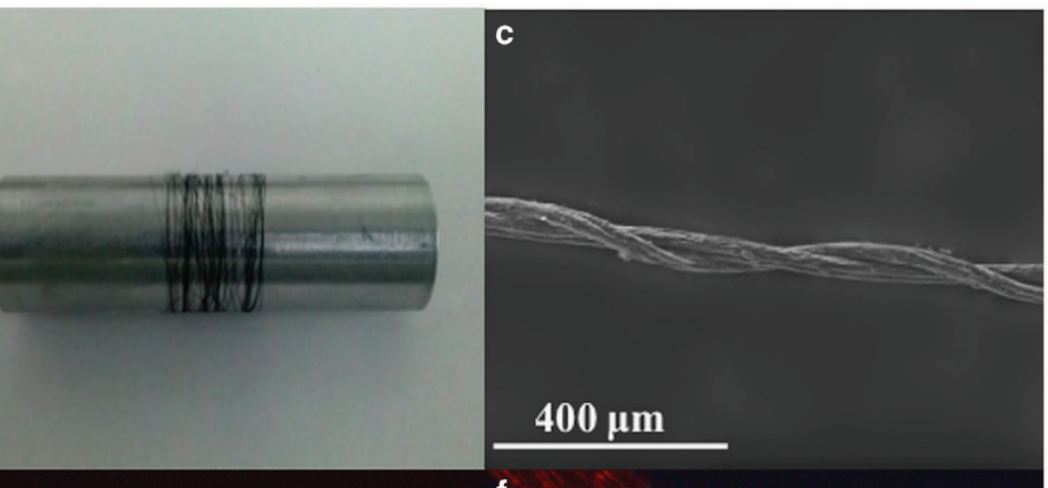

\section{$400 \mu \mathrm{m}$}

$\mathrm{f}$

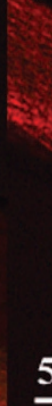

$\underline{50 \mu \mathrm{m}}$

$10 \mu \mathrm{m}$

Figure 3 (a) Wet spinning process in which four hybrid microfibers are spun at once. (b) Optical image of fibers from the $1 \mathrm{ml}$ of spinning solution wrapped around a $15 \mathrm{~cm}$ diameter steel stick. (c) Scanning electron microscopy (SEM) image of two hybrid microfibers twisted together. (d) Polarized optical microscopy (POM) image of a hybrid microfiber after extrusion from a syringe. (e) POM image of a hybrid microfiber that has been dried in air for $10 \mathrm{~s}$. (f) POM image of a hybrid microfiber under tension after drying completely in air.
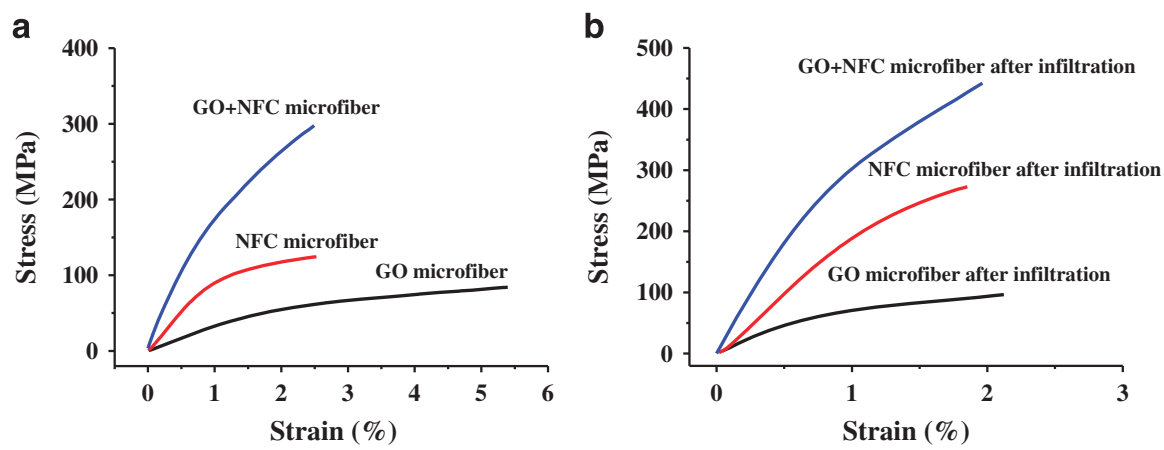

Figure 4 Typical stress-strain curves of GO microfibers, NFC microfibers, and GO-NFC hybrid microfibers before (a) and after (b) metal ion infiltration. GO, graphene oxide; NFC, nanofibrillated cellulose.

dramatically decreased from $\sim 80$ to $\sim 10 \mu \mathrm{m}$, and the fiber alignment increased, as illustrated in the polarized optical microscopy, which is a well-known method for fiber alignment evaluation (Figures $3 \mathrm{~d}-\mathrm{f}) .^{29}$ When the well-aligned hybrid microfiber was set parallel to the polarizer, only a dark image was obtained, as observed in Supplementary Figure S1c. A vivid fiber appears when the hybrid microfiber is rotated to an angle of $45^{\circ}$ with respect to the polarizer (Figure $3 \mathrm{f}$ ). The differences in texture between Figures $3 \mathrm{~d}-\mathrm{f}$ illustrate the enhanced alignment within the fiber as the drying process progresses.

Figure 4a presents the typical stress-strain curves of the GO microfibers, NFC microfibers and GO-NFC hybrid microfibers. The average elastic modulus and ultimate tensile strength (UTS) of the GO-NFC hybrid microfibers were $20.6 \pm 0.9 \mathrm{GPa}$ and $274.6 \pm 22.4$ $\mathrm{MPa}$, respectively, which are higher than those of both the NFC microfibers $(15.5 \pm 4.5 \mathrm{GPa}, 139.1 \pm 28.7 \mathrm{MPa})$ and the GO microfibers $(2.3 \pm 2 \mathrm{GPa}, 84.0 \pm 2.8 \mathrm{MPa})$ in our study. Although the mechanical performance of our GO-NFC hybrid microfiber is not superior to that reported in the literature, the synergistic effect of improving the mechanical properties of the fibers is clearly demonstrated.

To improve the mechanical properties of our GO-NFC hybrid microfibers, metal ions $\left(\mathrm{Ca}^{2+}\right)$ were utilized to introduce ionic bonding between the GO and the NFC by taking advantage of the oxygen and carboxylate groups from these two building blocks. ${ }^{20}$ Ionic bonding was introduced by infiltrating the hybrid microfibers in a 10 wt $\% \mathrm{CaCl}_{2}$ water solution. During the infiltration process, the fibers were rewetted and swelled. $\mathrm{Ca}^{2+}$ infiltrated within the fibers and formed ionic bonds with the building blocks after the drying process was complete. Figure $4 \mathrm{~b}$ depicts the typical stress-strain curves of the 


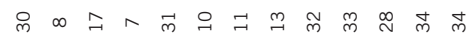

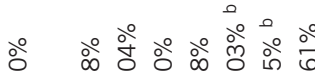$$
\text { ○워 }
$$

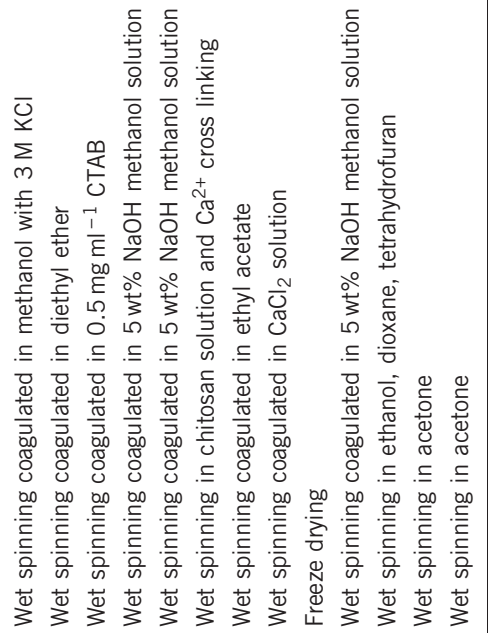

GO microfibers, NFC microfibers and GO-NFC hybrid microfibers after metal ion infiltration. After infiltration, the elastic modulus and the UST of the GO microfibers increased to $9.7 \mathrm{GPa}$ and $96.3 \mathrm{MPa}$, respectively. The NFC microfibers reached even higher elastic modulus $(20.7 \mathrm{GPa})$ and UST $(272 \mathrm{MPa})$ values. The infiltrated GO-NFC hybrid microfibers possessed an elastic modulus of $31.6 \pm 2.5 \mathrm{GPa}$ and a UTS of $416.6 \pm 25.8 \mathrm{MPa}$. Our best wet-spun infiltrated GO-NFC hybrid microfibers exhibited an elastic modulus of $34.1 \mathrm{GPa}$, a UTS of $442.4 \mathrm{MPa}$, and a tensile failure strain of $2.0 \%$. The mechanical properties of the GO microfibers and the NFC microfibers are listed in Table 1 and are compared with the values reported in the literature.

In terms of the elastic modulus, our infiltrated GO-NFC hybrid microfibers surpass pure NFC microfibers fabricated with longer lengths and higher aspect ratios $\left(22.5 \mathrm{GPa}^{28}\right.$ and $\left.23.6 \mathrm{GPa}^{34}\right)$, LC GO microfibers $(\sim 7.7 \mathrm{GPa}){ }^{7}$ stretched $\mathrm{Ca}^{2+}$ infiltrated GO microfibers $(6.3 \mathrm{GPa}$, after chemical reduction: $11.2 \mathrm{GPa}),{ }^{13} \mathrm{GO}$ microfibers coagulated from chitosan $(22.6 \mathrm{GPa})^{10}$ and biomimetic GO fiber $(20.9 \mathrm{GPa}),{ }^{12}$ but are inferior to the GO microfibers formed using large GO sheets (47 GPa). ${ }^{11}$ However, our infiltrated GO-NFC hybrid microfibers possess a high toughness of $4.9 \mathrm{MJ} \mathrm{m}^{-3}$ (note that the fiber in reference ${ }^{11}$ possesses a UTS of $214 \mathrm{MPa}$ and a strain of $0.6 \%$, which results in a lower toughness). The tensile strength of the infiltrated GO-NFC hybrid microfibers in this work is higher than that of wet-spun NFC microfibers $\left(275 \mathrm{MPa},{ }^{28} 402 \mathrm{MPa}^{34}\right)$, LC GO microfibers $(\sim 102 \mathrm{MPa}),{ }^{7}$ stretched $\mathrm{Ca}^{2+}$ infiltrated GO microfibers $(364 \mathrm{MPa}),{ }^{13}$ hydrothermally reduced GO microfibers (180 MPa, after annealing at $\left.800^{\circ} \mathrm{C}: 420 \mathrm{MPa}\right),{ }^{35}$ microfibers prepared from GO nanoribbons $\left(39.3 \mathrm{MPa}\right.$, after $1050{ }^{\circ} \mathrm{C}$ treatment: $\left.383 \mathrm{MPa}\right),{ }^{8} \mathrm{GO}$ microfibers formed using large GO sheets $(214 \mathrm{MPa})^{11}$ and is even compatible to that of chemically cross-linked GO fibers $(440 \pm 60$ $\mathrm{MPa}){ }^{16} \mathrm{~A}$ comparable tensile strength to our infiltrated hybrid microfiber is also exhibited by the GO microfibers prepared from very large GO sheets coagulated from chitosan (442 MPa); the average diameter of the GO sheet is $37 \mu \mathrm{m} .{ }^{10}$ The very large GO sheet size is the basis for the better mechanical properties of GO fibers. Our GO nanosheets exhibited an average diameter of $\sim 1.2 \mu \mathrm{m}$, which is much smaller than the sheets used by Jalili et al. ${ }^{10}(37 \mu \mathrm{m})$. We expect that future studies using larger GO sheets will allow us to create even stronger GO-NFC hybrid microfibers. The toughness of our fiber is $4.9 \mathrm{MJ} \mathrm{m}^{-3}\left(3.4 \mathrm{~J} \mathrm{~g}^{-1}\right)$. This value is higher than that of natural nacre $\left(1.8 \mathrm{MJ} \mathrm{m}^{-3}\right)^{36}$ and bone $\left(1.2 \mathrm{MJ} \mathrm{m}^{-3}\right)^{37}$ but lower than that of Kevlar (33-78 $\left.\mathrm{Jg}^{-1}\right)$ due to the nature of GO forming a brittle fiber. ${ }^{38,39}$ Wang et al. ${ }^{38}$ reported that extensive hydrogen bonding in the fiber restricts slippage of GO and thus leads to a high tensile strength but low strain to break and toughness.

Infiltrated GO-NFC hybrid microfibers exhibit a much higher elastic modulus and tensile strength compared with GO-only or NFConly microfibers, the same phenomenon in un-infiltrated fibers. This synergistic effect is also observed in 1D carbon nanotube (CNT)-2D GO hybrid fibers and 1D CNT-2D reduced GO hybrid fibers. ${ }^{38,39}$ The underlying hypothesis is that 1D flexible NFC fibers have good interfacial contact with 2D GO nanosheets, and NFC provides extra bonding options between the GO nanosheets. 1D NFC flexible fibers can act as 'lines' to 'weave and wrap' 2D nanosheets together. 2D GO nanosheets can create a bridge between the neighboring NFC fibers, providing extra bonding sites between 1D NFC fibers over a long distance. To understand the synergistic effect between the 2D GO nanosheets and the 1D NFC, we examined the structural characteristics of various types of fibers because we expected the inter-sheet bonding to have a considerable effect on the fiber morphology. After the drying process under tension, the GO-NFC hybrid microfibers 
exhibited improved mechanical performance due to the alignment of the NFC along the fiber direction as well as the circularity of the fiber cross section. The addition of the NFC within the fiber yielded a smoother surface and a higher packing density for the non-infiltrated GO-NFC hybrid microfibers. The infiltration of metal ions further improved the surface smoothness and packing density for the hybrid microfibers. These conclusions are evident and verified in the schematics presented in Figures $5 \mathrm{a}-\mathrm{c}$ as well as the scanning electron microscope characterization (Figures $5 \mathrm{~d}-\mathrm{f}$ ). The well-ordered, denselystacked GO nanosheets in the middle of the fiber cross-section signify good alignment throughout the fiber (Figures 5g-i). We observed that metal ion infiltration led to more densely packed and well-ordered GO nanosheets within the resulting GO-NFC hybrid microfibers (Figures $5 j-1)$. The prior observations suggest that the surface smoothness and packing density are strongly dependent on the bonding between the GO nanosheets. This finding directly correlates with the enhanced mechanical performance of the resulting GO-NFC hybrid microfibers because the inter-sheet sliding is the dominant microscopic deformation and failure mechanism of these fibers under tension. In other words, when the inter-sheet bonding density is increased, the surface a

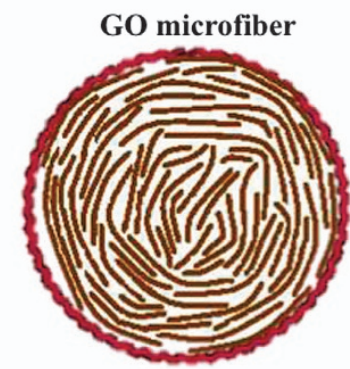

b

GO-NFC hybrid microfiber

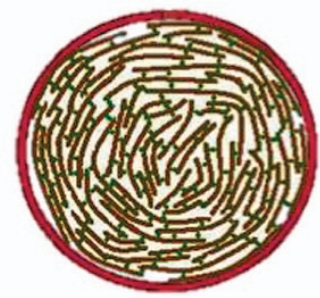

C
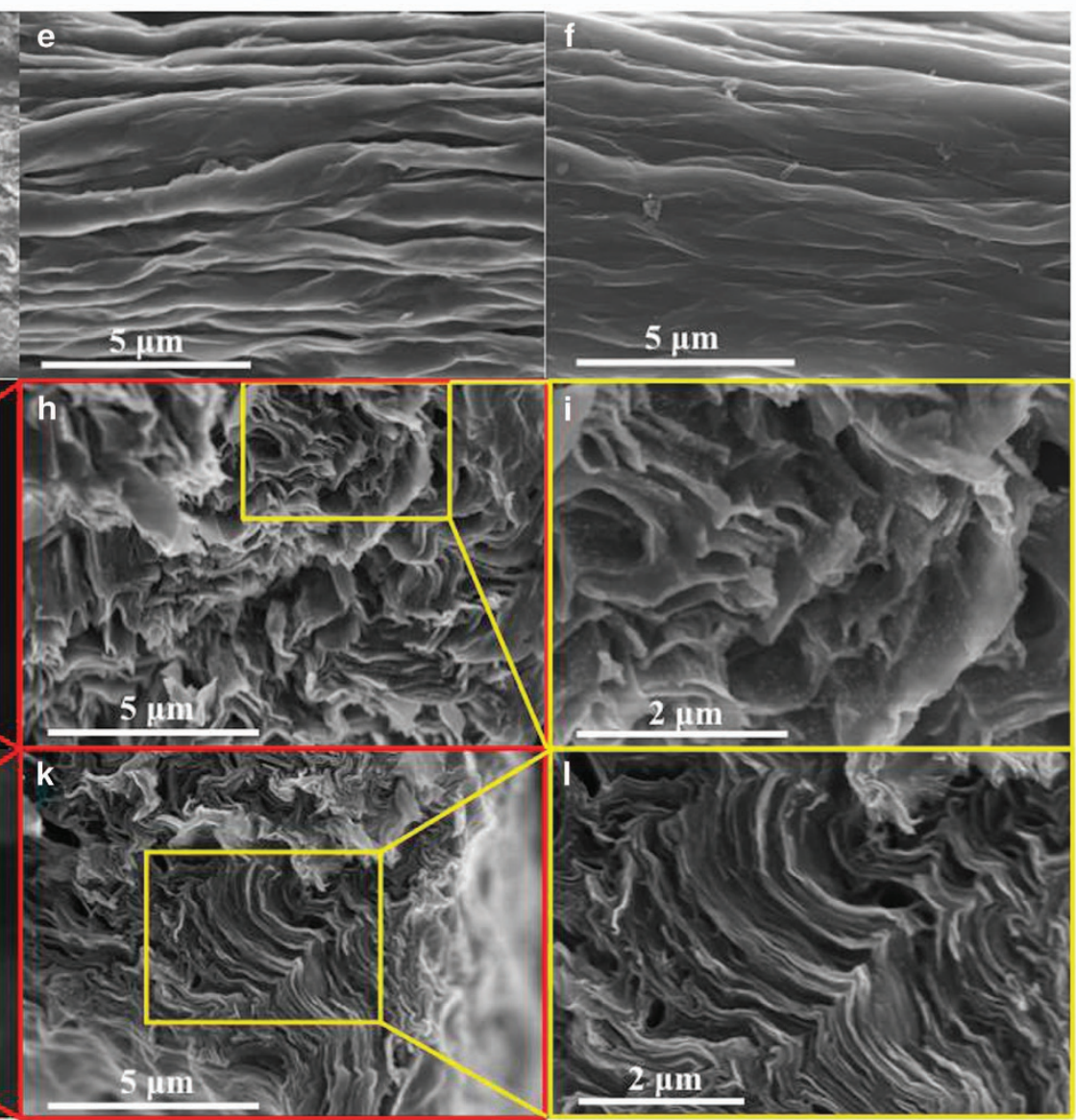

Figure 5 Schematic illustration of the cross section of (a) a GO microfiber, (b) a GO-NFC hybrid microfiber and (c) a GO-NFC hybrid microfiber after infiltration. The brown lines represent the GO nanosheet and the green dots represent NFC. The GO microfiber exhibits loose GO nanosheet packing and a corrugated rough surface. When NFC is added, the GO-NFC hybrid microfiber possesses a smoother surface and denser packing. After infiltration in the CaCl ${ }_{2}$ solution, the fiber surface is even smoother and possesses even denser building block packing compared with the GO-NFC hybrid microfiber. SEM images of the surface morphology of (d) the porous GO microfiber, (e) the GO-NFC hybrid microfiber and (f) the GO-NFC hybrid microfiber after metal ion infiltration from the $\mathrm{CaCl}_{2}$ solution. After NFC is added, the surface becomes dense and smooth; however, after infiltration, the fiber becomes exceptionally dense and smooth. SEM images displaying the cross section of ( $\mathbf{g}-\mathbf{i})$ the GO-NFC hybrid microfiber before and (j-I) after $\mathrm{CaCl}_{2}$ infiltration. $\mathrm{GO}$, graphene oxide; NFC, nanofibrillated cellulose; SEM, scanning electron microscopy. 
of the fiber will be smoother, there will be a higher packing density, and the GO-NFC hybrid microfibers will be stronger and tougher.

To validate the above hypothesis and gain mechanistic insight into the strength of the GO-NFC hybrid microfibers, we performed systematic molecular dynamics modeling to reveal the fundamental deformation and failure mechanisms of the three types of microfibers fabricated in this study. Given the ultra-strong GO sheets, the tensile failure of the GO microfibers stemmed from the inter-GO-sheet sliding instead of the individual GO sheets fracturing. In a pure GO microfiber, hydrogen bonding can form between neighboring GO sheets only when one hydroxyl group from one GO sheet comes in close contact with another hydroxyl group (or oxygen functional group) from another GO sheet (that is, $-\mathrm{OH}$ to $-\mathrm{OH}$ or $-\mathrm{OH}$ to $-\mathrm{O}-$; Figure 6a). Conversely, in a GO-NFC hybrid microfiber, the abundant hydroxyl groups in NFC can significantly increase the number of hydrogen bonds between the neighboring GO sheets due to the availability of $-\mathrm{O}-$ to $-\mathrm{O}-$ bonding (Figure $6 \mathrm{~b}$ ). Owing to the nature of hydrogen bonding, the inter-GO-sheet sliding involves a cascade of formation, breaking and reformation of hydrogen bonding (Figures $6 \mathrm{c}$ and d). Therefore, the hydrogen bond density has an important role in the mechanical properties of these microfibers (for example, tensile strength and toughness). To provide quantitative evidence of the effect of hydrogen bond density, we performed molecular dynamics modeling to simulate the representative inter-GO-sheet sliding events leading up to the tensile failure of a GO microfiber (Figure 6c) and a GONFC hybrid microfiber (Figure 6d). Figure 6e plots the force needed to drive the inter-GO-sheet sliding as a function of the sliding displacement for both fibers. The fluctuation of the two curves reflects the breaking and reformation of the hydrogen bonds as the sliding proceeds. It is evident that significantly increasing the amount of hydrogen bonds from the NFC molecular chains sandwiched in between GO sheets leads to a nearly twofold increase of the peak force that drives the inter-GO-sheet sliding compared with the pure GO sheets.

This increase in peak force, which causes hydrogen bond breaking, offers a mechanistic understanding of the enhanced tensile strength of the GO-NFC hybrid microfibers relative to the GO microfibers at the atomic scale. The area underneath the curves in Figure 6e represents the fracture energy (that is, the work performed by the sliding force) needed to drive the inter-GO-sheet sliding. The increased fracture
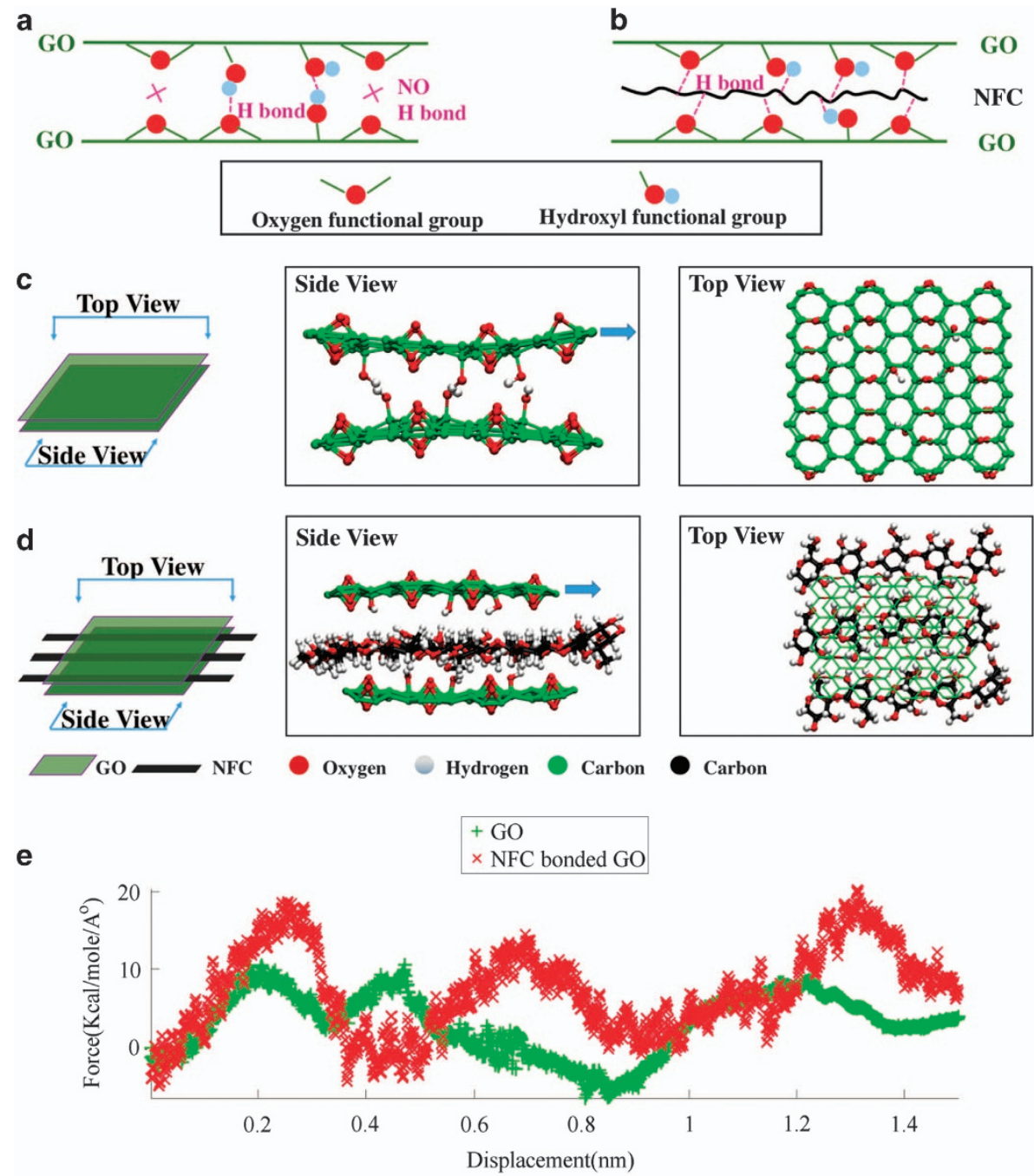

Figure $6(\mathbf{a}, \mathbf{b})$ Schematic illustrations showing the role of NFC in providing additional bonding options between GO sheets. (c, d) Molecular dynamics models for GO sheets without and with NFC. The insets show typical equilibrium structural configurations from different perspectives before the top sheet slides. (e) Comparison of the sliding force as a function of sliding displacement between the two cases (i.e., with and without NFC). GO, graphene oxide; NFC, nanofibrillated cellulose. 
energy due to a higher hydrogen bond density provided by NFC explains the enhanced fracture toughness exhibited by our GO-NFC hybrid microfibers. Additional simulations (Supplementary Figure S2) reveal that such enhancements in the peak force to drive inter-sheet sliding and the associated fracture energy in a GO-NFC hybrid microfiber are even more significant when compared with a GO microfiber in the case of GO nanosheets without hydroxyl groups. The results also suggest that a smoother surface morphology of the GO sheets in a GO-NFC hybrid microfiber can facilitate more facile formation of inter-sheet hydrogen bonds via NFC, which leads to a microfiber with higher tensile strength and fracture toughness. This finding agrees with our experimental observations of the GO-NFC hybrid microfiber after $\mathrm{CaCl}_{2}$ infiltration (Figure 5).

The enhanced mechanical properties of the GO-NFC hybrid microfibers compared with the NFC microfibers can be understood as follows. In our samples, the length of the NFC chains is smaller than the feature size of the GO nanosheets. Therefore, in the GO-NFC hybrid microfibers, a GO sheet (2D) can link several NFC chains (1D) together via hydrogen bonding in both the aligned microfiber direction and in the transverse direction, providing extra bonding sites between NFC chains over a long distance (Figures 7a and c). By contrast, in the NFC microfibers, a rather small length of NFC chains can be linked together via other NFC chains (Figures $7 \mathrm{~b}$ and $d$ ). To further clarify the quantitative effect of the GO sheets, we performed molecular dynamics simulations of inter-NFC-sliding in these two cases, as illustrated in Figures $7 \mathrm{c}$ and $\mathrm{d}$. As shown in Figure $7 \mathrm{e}$, the existence of GO sheets linking together the neighboring NFC chains can effectively increase both the peak force to drive the inter-NFC sliding as well as the associated fracture energy relative to the case of pure NFC microfibers. These molecular dynamics simulation results offer a mechanistic understanding of the enhanced mechanical properties of the GO-NFC hybrid microfibers compared with the NFC microfibers (Figure 4).

To further prove the synergetic interaction between the $2 \mathrm{D}$ GO nanosheets and 1D NFC, we also prepared a CNT-NFC microfiber (weight ratio of CNT:NFC is 1:1). Details can be found in the supporting materials. Supplementary Figure S3 presents a typical stress-strain curve of the CNT-NFC microfiber. The average tensile strength of the CNT-NFC microfiber is $\sim 200 \mathrm{MPa}$, higher than that of the NFC microfiber $(139.1 \pm 28.7 \mathrm{MPa})$ but lower than that of the GO-NFC microfiber $(274.6 \pm 22.4 \mathrm{MPa})$. This finding helps us to confirm that the synergetic interaction between the $2 \mathrm{D}$ a

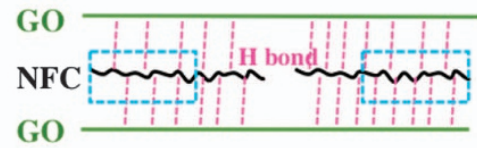

b

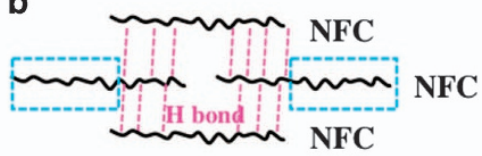

C

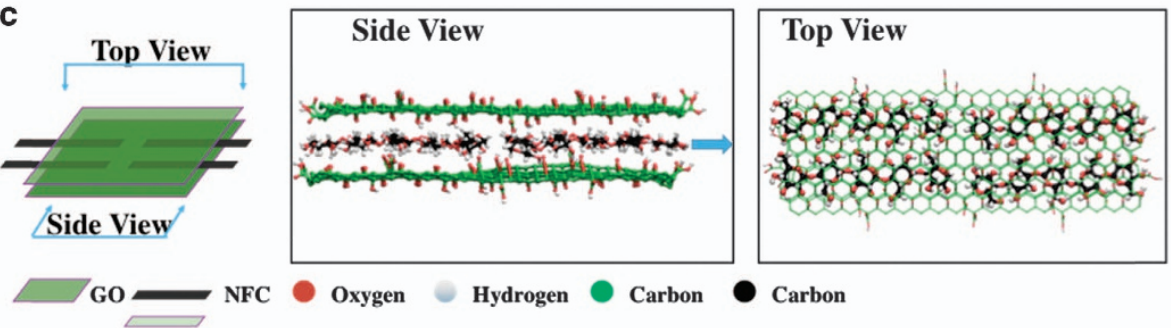

d

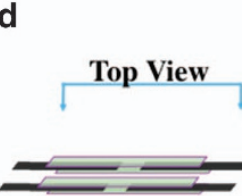

Side View
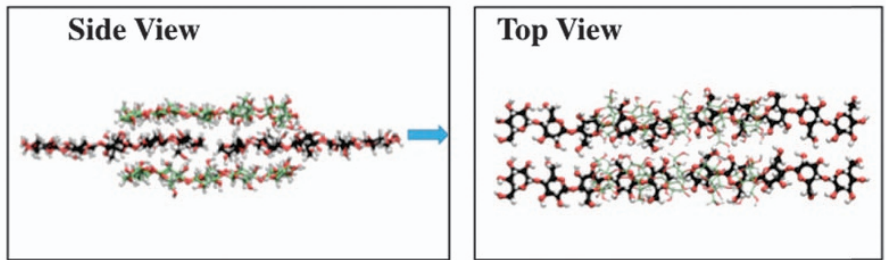

e

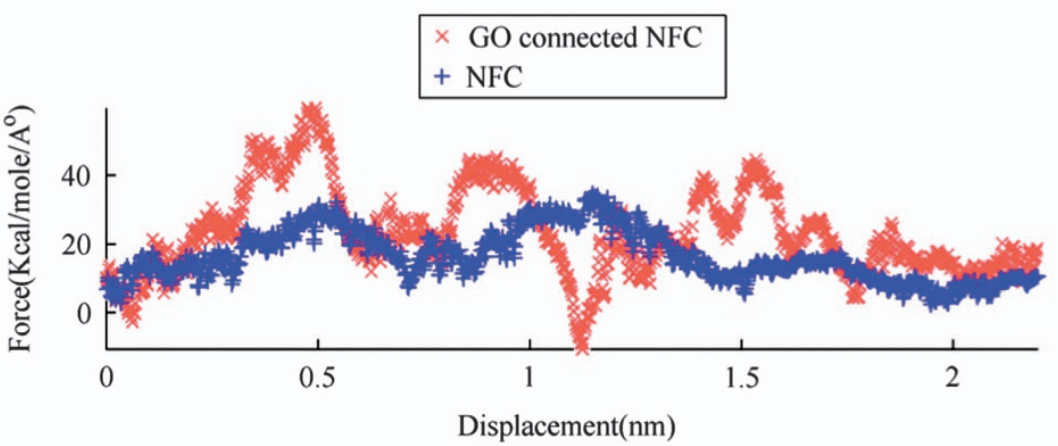

Figure 7 (a, b) Schematic illustrations demonstrating the role that GO has in providing additional bonding sites (dashed boxes) between the NFC chains. (c-d) Molecular dynamics models for NFC chains with and without GO sheets. The insets show typical equilibrium structural configurations from different perspectives before the right NFC chains slide out. (e) Comparison of the sliding force as a function of sliding displacement between the above two cases (i.e., with and without GO sheets). GO, graphene oxide; NFC, nanofibrillated cellulose. 
GO and 1D NFC is the key factor to the enhanced mechanical performance.

\section{CONCLUSIONS}

Well-aligned, strong microfibers were successfully fabricated by wet spinning a LC solution consisting of 2D GO nanosheets with 1D NFC. The major finding is that well-aligned hybrid microfibers are much stronger than the microfibers composed of 1D NFC or 2D GO alone. Molecular dynamics simulations reveal that the synergetic interaction between the 2D GO and 1D NFC is the key factor contributing to the enhanced mechanical performance. 1D flexible NFC fibers have good interfacial contact with 2D GO nanosheets, and can provide extra bonding options between the GO nanosheets. In addition, 1D NFC flexible fibers can act as 'lines' to 'weave and wrap' 2D nanosheets together. 2D GO nanosheets create a bridge between the neighboring NFC fibers, providing extra bonding sites between 1D NFC fibers over a long distance. After infiltrating $\mathrm{Ca}^{2+}$ within the GO-NFC hybrid microfibers, improvements in the mechanical performance were observed; in particular, fibers with a UTS and an elastic modulus of $442.4 \mathrm{MPa}$ and $34.1 \mathrm{GPa}$, respectively, were obtained. Note that the infiltrated GO-NFC microfibers were also lightweight in nature. The low-cost, earth-abundant building blocks used in this study satisfy the economic requirement to produce mechanically strong fibers for potential commercial use. We expect that further studies using high-quality building materials, for example, larger GO nanosheets, an improved fabrication process and infiltration of other metal ions may enable us to create even stronger and tougher microfibers. Additionally, the mechanistic finding of the synergetic interactions between 2D and 1D building blocks can be potentially applicable to the design of high-performance microfibers composed of other materials.

\section{CONFLICT OF INTEREST}

The authors declare no conflict of interest.

\section{ACKNOWLEDGEMENTS}

LH and TL acknowledge the support of the National Science Foundation (Grant Number: 1362256). TL and SZ acknowledge the support received by the National Science Foundation (Grant Numbers: 1069076 and 1129826). SZ acknowledges the support of the Clark School Future Faculty Program at the University of Maryland. YL and HD would like to acknowledge the support received by the National Natural Science Foundation of China (Grant Number: 31470599) and the Priority Academic Program Development of Jiangsu Higher Education Institutions (PAPD). We would also like to acknowledge Professor LJ Martinez-Miranda for helping us to image the fibers using the polarized microscope.

1 Siro, I. \& Plackett, D. Microfibrillated cellulose and new nanocomposite materials: a review. Cellulose 17, 459-494 (2010).

2 Moon, R. J., Martini, A., Nairn, J., Simonsen, J. \& Youngblood, J. Cellulose nanomaterials review: structure, properties and nanocomposites. Chem. Soc. Rev. 40, 3941-3994 (2011).

3 Wu, C. N., Saito, T., Fujisawa, S., Fukuzumi, H. \& Isogai, A. Ultrastrong and high gas-barrier nanocellulose/clay-layered composites. Biomacromolecules 13 , 1927-1932 (2012).

4 Uddin, A. J., Araki, J. \& Gotoh, Y. Toward "strong" green nanocomposites: polyvinyl alcohol reinforced with extremely oriented cellulose whiskers. Biomacromolecules $\mathbf{1 2}$, 617-624 (2011).

5 Xu, Z. \& Gao, C. Graphene in macroscopic order: liquid crystals and wet-spun fibers. Acc. Chem. Res. 47, 1267-1276 (2014).

6 Stankovich, S., Dikin, D. A., Dommett, G. H. B., Kohlhaas, K. M., Zimney, E. J., Stach, E. A., Piner, R. D., Nguyen, S. T. \& Ruoff, R. S. Graphene-based composite materials. Nature 442, 282-286 (2006)
7 Xu, Z. \& Gao, C. Graphene chiral liquid crystals and macroscopic assembled fibres. Nat. Commun. 2, 571 (2011).

8 Xiang, C. S., Behabtu, N., Liu, Y. D., Chae, H. G., Young, C. C., Genorio, B. Tsentalovich, D. E., Zhang, C. G., Kosynkin, D. V., Lomeda, J. R., Hwang, C. C., Kumar, S., Pasquali, M. \& Tour, J. M. Graphene nanoribbons as an advanced precursor for making carbon fiber. ACS Nano 7, 1628-1637 (2013).

9 Jang, E. Y., Carretero-Gonzalez, J., Choi, A., Kim, W. J., Kozlov, M. E., Kim, T., Kang, T. J., Baek, S. J., Kim, D. W., Park, Y. W., Baughman, R. H. \& Kim, Y. H. Fibers of reduced graphene oxide nanoribbons. Nanotechnology 23, 235601 (2012).

10 Jalili, R., Aboutalebi, S. H., Esrafilzadeh, D., Shepherd, R. L., Chen, J., Aminorroaya-Yamini, S., Konstantinov, K., Minett, A. I., Razal, J. M. \& Wallace, G. G. Scalable one-step wet-spinning of graphene fibers and yarns from liquid crystalline dispersions of graphene oxide: towards multifunctional textiles. Adv. Funct. Mater. 23 5345-5354 (2013).

11 Xiang, C. S., Young, C. C., Wang, X., Yan, Z., Hwang, C. C... Cerioti, G., Lin, J., Kono, J., Pasquali, M. \& Tour, J. M. Large flake graphene oxide fibers with unconventional $100 \%$ knot efficiency and highly aligned small flake graphene oxide fibers. Adv. Mater. 25, $4592-4597$ (2013).

$12 \mathrm{Hu}$, X. Z., Xu, Z., Liu, Z. \& Gao, C. Liquid crystal self-templating approach to ultrastrong and tough biomimic composites. Sci. Rep. 3, 2374 (2013).

$13 \mathrm{Xu}$, Z., Sun, H. Y., Zhao, X. L. \& Gao, C. Ultrastrong fibers assembled from giant graphene oxide sheets. Adv. Mater. 25, 188-193 (2013).

$14 \mathrm{Kou}, \mathrm{L}$. \& Gao, C. Bioinspired design and macroscopic assembly of poly(vinyl alcohol)coated graphene into kilometers-long fibers. Nanoscale 5, 4370-4378 (2013).

$15 \mathrm{Hu}$, X. Z., Xu, Z. \& Gao, C. Multifunctional, supramolecular, continuous artificial nacre fibres. Sci. Rep. 2, 767 (2012).

16 Zhao, X., Xu, Z., Zheng, B. \& Gao, C. Macroscopic assembled, ultrastrong and H2SO4-resistant fibres of polymer-grafted graphene oxide. Sci. Rep. 3, 3164 (2013).

17 Cong, H.P., Ren, X. C., Wang, P. \& Yu, S. H. Wet-spinning assembly of continuous, neat, and macroscopic graphene fibers. Sci. Rep. 2, 613 (2012).

18 Li, Y. Y., Zhu, H. L., Gu, H. B., Dai, H. Q., Fang, Z. Q., Weadock, N. J., Guo, Z. H. \& $\mathrm{Hu}, \mathrm{L}$. B. Strong transparent magnetic nanopaper prepared by immobilization of $\mathrm{Fe}_{3} \mathrm{O}_{4}$ nanoparticles in a nanofibrillated cellulose network. J. Mater. Chem. A $\mathbf{1}$, 15278-15283 (2013).

19 Marcano, D. C., Kosynkin, D. V., Berlin, J. M., Sinitskii, A., Sun, Z. Z., Slesarev, A., Alemany, L. B., Lu, W. \& Tour, J. M. Improved synthesis of graphene oxide. ACS Nano 4, 4806-4814 (2010).

20 Mattsson, T., Lane, J., Cochrane, K., Desjarlais, M., Thompson, A., Pierce, F. \& Grest, G. First-principles and classical molecular dynamics simulation of shocked polymers. Phys. Rev. B 81, 054103 (2010).

21 Plimpton, S. Fast parallel algorithms for short-range molecular-dynamics. J. Comput. Phys. 117, 1-19 (1995).

22 Compton, O., Cranford, S., Putz, K., An, Z., Brinson, L., Buehler, M. \& Nguyen, S. Tuning the mechanical properties of graphene oxide paper and its associated polymer nanocomposites by controlling cooperative intersheet hydrogen bonding. ACS Nano 6, 2008-2019 (2012).

23 Jiang, F. \& Hsieh, Y. L. Chemically and mechanically isolated nanocellulose and their self-assembled structures. Carbohydr. Polym. 95, 32-40 (2013).

24 Chae, H. G. \& Kumar, S. Materials science-making strong fibers. Science $\mathbf{3 1 9}$, 908-909 (2008)

25 Sun, X. M., Chen, T., Yang, Z. B. \& Peng, H. S. The alignment of carbon nanotubes: an effective route to extend their excellent properties to macroscopic scale. Acc. Chem. Res. 46, 539-549 (2013).

26 Habibi, Y., Lucia, L. A. \& Rojas, O. J. Cellulose nanocrystals: chemistry, self-assembly, and applications. Chem. Rev. 110, 3479-3500 (2010).

27 Jalili, R., Aboutalebi, S. H., Esrafilzadeh, D., Konstantinov, K., Moulton, S. E. Razal, J. M. \& Wallace, G. G. Organic solvent-based graphene oxide liquid crystals: a facile route toward the next generation of self-assembled layer-by-layer multifunctional 3D architectures. ACS Nano 7, 3981-3990 (2013).

28 Walther, A., Timonen, J. V. I., Diez, I., Laukkanen, A. \& Ikkala, O. Multifunctional highperformance biofibers based on wet-extrusion of renewable native cellulose nanofibrils. Adv. Mater. 23, 2924-2928 (2011).

29 Zhang, S. J., Koziol, K. K. K., Kinloch, I. A. \& Windle, A. H. Macroscopic fibers of well-aligned carbon nanotubes by wet spinning. Small 4, 1217-1222 (2008).

30 Zhao, Y., Jiang, C. C. Hu, C. G., Dong, Z. L., Xue, J. L., Meng, Y. N., Zheng, N. Chen, P. W. \& Qu, L. T. Large-scale spinning assembly of neat, morphology-defined, graphene-based hollow fibers. ACS Nano 7, 2406-2412 (2013).

31 Chen, L., He, Y.L., Chai, S.G., Qiang, H., Chen, F. \& Fu, Q. Toward high performance graphene fibers. Nanoscale 5, 5809-5815 (2013).

32 Xu, Z., Zhang, Y., Li, P. G. \& Gao, C. Strong, conductive, lightweight, neat graphene aerogel fibers with aligned pores. ACS Nano 6, 7103-7113 (2012).

33 Yang, Z. B., Sun, H., Chen, T., Quu, L. B., Luo, Y. F. \& Peng, H. S. Photovoltaic wire derived from a graphene composite fiber achieving an $8.45 \%$ energy conversion efficiency. Angew. Chem. Int. Ed. 52, 7545-7548 (2013).

34 Iwamoto, S., Isogai, A. \& Iwata, T. Structure and mechanical properties of wet-spun fibers made from natural cellulose nanofibers. Biomacromolecules 12 , 831-836 (2011).

35 Dong, Z. L., Jiang, C. C., Cheng, H. H., Zhao, Y., Shi, G. Q., Jiang, L. \& Qu, L. T. Facile fabrication of light, flexible and multifunctional graphene fibers. Adv. Mater. 24, 1856-1861 (2012). 
36 Wang, R. Z., Suo, Z., Evans, A. G., Yao, N. \& Aksay, I. A. Deformation mechanisms in nacre. J. Mater. Res. 16, 2485-2493 (2001).

37 Rho, J. Y., Spearing, L. K. \& Zioupos, P. Mechanical properties and the hierarchical structure of bone. Med. Eng. Phys. 20, 92-102 (1998).

38 Wang, R. R., Sun, J., Gao, L., Xu, C. H. \& Zhang, J. Fibrous nanocomposites of carbon nanotubes and graphene-oxide with synergetic mechanical and actuative performanc. Chem. Commun. 47, 8650-8652 (2011).

39 Shin, M. K., Lee, B., Kim, S. H., Lee, J. A., Spinks, G. M., Gambhir, S., Wallace, G. G., Kozlov, M. E., Baughman, R. H. \& Kim, S. J. Synergistic toughening of composite fibres by self-alignment of reduced graphene oxide and carbon nanotubes. Nat. Commun. 3 , 650 (2012). (c) (1)(9) This work is licensed under a Creative Commons Attribution-NonCommercial-ShareAlike $\quad 4.0$ International License. The images or other third party material in this article are included in the article's Creative Commons license, unless indicated otherwise in the credit line; if the material is not included under the Creative Commons license, users will need to obtain permission from the license holder to reproduce the material. To view a copy of this license, visit http://creativecommons.org/licenses/ by-nc-sa/4.0/

Supplementary Information accompanies the paper on the NPG Asia Materials website (http://www.nature.com/am) 